exchange interaction. For example, the 2 e configuration shown in Fig. 1 has a classic spin structure that consists of a singlet and triplet energy difference of about $5 \mathrm{meV}$. In contrast, the electronhole exchange between an unpaired electron and an unpaired hole is an order of magnitude less. The electron-hole exchange splittings, though just barely observable in the data ${ }^{1}$, are important in the identifications of the spectral lines.

Being physicists, we are pleased when we reach a simple intuitive understanding of our observations; but as researchers, the unexpected excite us. As Ediger et al. demonstrate through a detailed comparison of experiment and theory, the unexpected comes in many ways. One source (among many) of counterintuitive splittings arises from the fact that the spin state of the hole is not as simple as we like to think. Being in the valence band of the semiconductor, the hole derives from $p$ states of the atoms in the lattice. Thus it is not a simple spin-1/2 particle like the electron, but rather has a strong spin-orbit character. The simplest example of unexpected splittings is found in the case of the $2 \mathrm{~h}$ state with each hole in a different orbital. In contrast to the 2e case, the singlet-triplet symmetry is broken and three spectral lines are found instead of two. It is but one of many intriguing differences that come to light in the spectra.

The ability to measure the quantum states of custom-engineered individual dots is enabling rapid scientific progress in this field. The paper of Ediger et al. represents a broad research thrust aimed at understanding the physics of quantum dots through spectroscopy. Dots also form artificial molecules. There, the combination of spin exchange and coherent tunnelling leads to very rich patterns of fine structure ${ }^{3}$, again with pleasing analogues in the physics of molecules but with features unique to solid-state structures. Quantum dots also can be grown within optical cavities to induce strong coupling with a cavity photon ${ }^{4}$. In a strong laser field a single dot shows Autler-Towns splittings and
Mollow triplets, classic examples of coherent optical experiments ${ }^{5}$. In fact many of the techniques of quantum optics that have been developed using atoms are now being performed on dots.

Besides this optical point of view, a large and sophisticated transport community is also studying the quantum $\operatorname{dot}^{6}$. Moreover, the opportunities go beyond pure scientific curiosity and discovery. The ability to engineer and optically probe individual quantum dots leads to the ability to control their quantum state ${ }^{2}$, which brings this research area into the exciting world of quantum information science and technology. No matter how you look at it, the quantum dot is fundamental and exciting - both in concept and in reality.

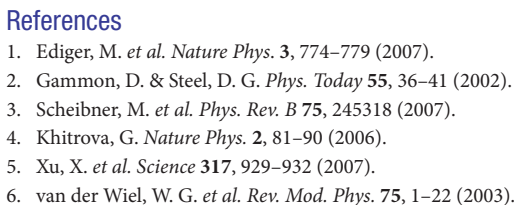

\title{
AERODYNAMICS
}

\section{Four wings good}

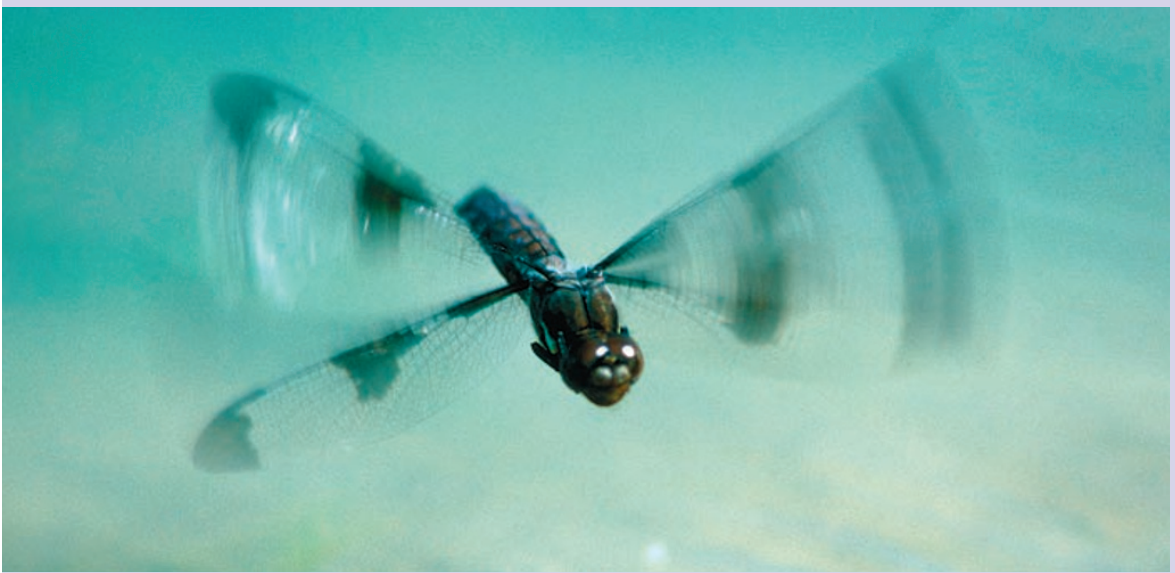

Dragonflies can out-manoeuvre other flying insects - a great benefit given their diet of mosquitoes and other flying pests. They are unique among four-winged flyers: their two sets of wings, known as the forewings and hindwings, can move independently of each other. When taking off from standstill, the wings beat in phase, but while hovering, they are out of phase. Despite many studies using photography, film and computer simulations, the wing interactions have yet to be fully understood. To this end, Z. Jane Wang and David Russell have used a high-speed camera to record the tethered flight of the dragonfly Libellula Pulchella (Phys. Rev.

Lett. 99, 148101; 2007).

The authors painted three points on each wing so that, as well as wing velocity, they also recorded the threedimensional motion of the hovering wings - including the time-dependent angle of attack. They then entered a twodimensional projection of their data into their calculation of the aerodynamic force and power as a function of the phase lag between the forewings and hindwings, which are close enough to interact hydrodynamically. Unsurprisingly, the vertical force and power are maximal when the wings are in phase, as at takeoff. But the force drops quickly with increasing phase difference, whereas the power falls more slowly, reaching a minimum at $160^{\circ}$. The phase difference helps reduce the required power while generating the minimal force required to balance the weight. Moreover, the relative flatness of the power minimum for hovering, between $100^{\circ}$ and $220^{\circ}$, means that precise control of the phase is not necessary.

For a microscopic explanation, Wang and Russell use the analogy of two moving cylinders. For cylinders moving in opposite directions, the net drag on each is reduced. But the reduced drag points in opposite directions and cancel, so the net force is nearly the same in hovering mode. However, the net power is reduced, as it is an additive effect.

Such a detailed understanding of the wing-wing interactions not only helps us appreciate the flight of the dragonfly, but it may also lead to improved aircraft designs for increased stability and energy efficiency. 